\section{Check Out the AATS Week Video}

Learn more about the exciting program planned for the AATS Aortic Symposium and 2016 Annual Meeting.

Check out the AATS Week Video: https://www.youtube.com/watch? $\mathrm{v}=$ AdIdZi4ZniE

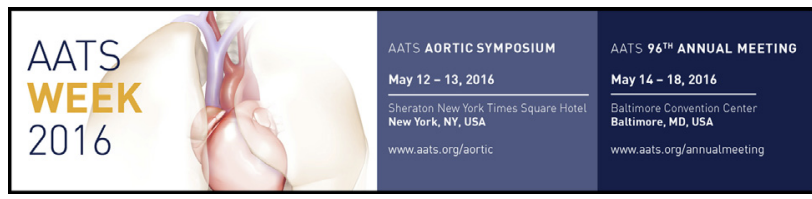

\section{View AATS Surgical Patient Safety Course Program/Register Today!}

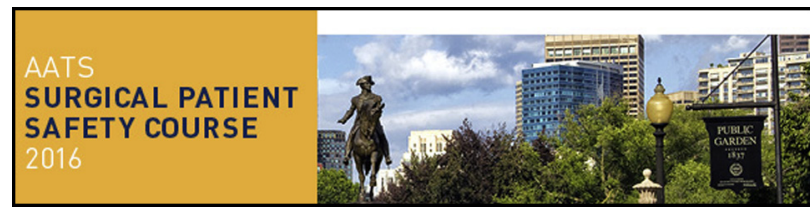

June 24-25, 2016

Renaissance Boston Waterfront Hotel

Boston, MA

\section{Co-Directors}

Thoralf M. Sundt III, MD

Steven Yule, $\mathrm{PhD}$

\section{Program Committee}

David J. Bunnell, PA-C, APACVS

David C. Fitzgerald, CCP, AMSECT

Jake Jaquiss, MD

M. Blair Marshall, MD

Shannon Pengel, RN

Kenneth Shann, CCP, LP, AMSECT

Marco Zenati, MD

Improving patient care remains a constant mission for all members of the surgical team. The AATS Surgical Patient Safety Course has been designed to promote a culture of safety and reduce preventable patient harm by engaging, educating and equipping members of the cardiothoracic surgical team in the multidimensional approach to patient safety.

The course, which features didactic and interactive presentations and focused workshops, will provide attendees with essential patient safety knowledge, skills, and attitudes aimed at improving care in the present and future.

Preliminary Program Now Online: http://aats.org/patient safety/abstracts/2015-Program.cgi.

The Patient Safety program includes team training and simulation experiences.

\section{Surgical Team Registration}

Register three or more members from the same institution and receive one complimentary Health care Professional registration. We encourage you to send the entire team to Boston this June for this important course.

Register Your Team Before May 23, 2016, and Save: www.aats.org/patientsafety/registration.cgi.

\section{The AATS Graham Foundation}

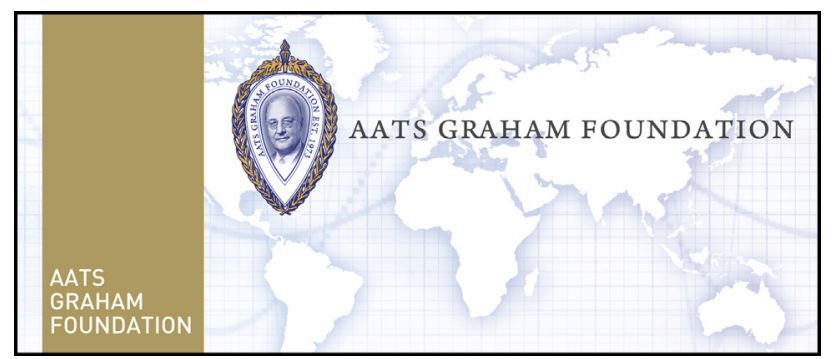

\section{Graham Foundation News}

\section{Congratulations to Our 2015 Awardees}

Evarts A. Graham Traveling Fellowship

Dr Konrad Hoetzenecker of the University of Vienna was selected as the 2016-2017 AATS Graham Traveling Fellow.
Dr Hoetzenecker's Fellowship year will begin at the 2016 AATS Annual Meeting and will take place at Toronto General Hospital in Toronto, ON, Canada under the primary sponsorship of Dr Thomas K. Waddell.

\section{Third Robert E. Gross Research Scholarship}

Dr Matthew D. Taylor was selected by the AATS as the Third Robert E. Gross Research Scholarship.

Dr Taylor's two-year research scholarship at Penn State Milton S. Hershey Medical Center with begin in July 2016. Advanced Valve Disease Educational Fellowship Sponsored by Medtronic

Govind Chetty, MD — University Hospital of Wales, United Kingdom

Andrea Colli, MD - University of Padua, Italy

Evaldas Girdauskas, MD—Central Hospital Bad Berka, Germany 
Francisco Guzmán, MD_Hospital Español De Mendoza, Argentina

Paradorn Jetwanna, MD_-Suratthani Hospital, Thailand

Vasily Kaleda, MD — Kuban State Medical University, Russia 2015 Fellows for Advanced Minimally Invasive Thoracic Surgery-Sponsored by Ethicon

Ki Wan Kim, MD_NorthShore University Health System, Evanston, IL

John F. Lazar, MD_Pinnacle Health Cardiovascular Institute, Harrisburg, PA

Gholam R. Mohammadzadeh, MD—Los Robles Hospital, Thousand Oaks, CA

James L. Cox Fellowship in Atrial Fibrillation SurgerySponsored by AtriCure

Safak Alpat, MD-Hacettepe University School of Medicine, Department of Cardiovascular Surgery, Turkey

Atsushi Hiromoto, MD-M.M. Amosov National Institute of Cardiovascular Surgery, Japan

Dong Seop Jeong, MD_-Samsung Medical Center, Republic of Korea

Paradorn Jetwanna, MD_Suratthani Hospital, Thailand

Eske Sindby, MD_Aalborg University Hospital, Denmark

Zhiwei Xu, MD_Department of Cardiothoracic Surgery, Xinhua Hospital, Shanghai Jiao Tong

AAATS Graham Foundation Academic Excellence Award

Dr Calogera Pisano

Best Work in Progress Presentation on Innovation and Scientific Research

29th EACTS Annual Meeting-Amsterdam, The Netherlands

Dr Lieven Depypere

Best Scientific Contribution on Esophageal Disease 23rd European Conference on General Thoracic SurgeryLisbon, Portugal

2015 Every Heartbeat Valve Matters Fellows-Sponsored by Edwards Lifesciences

Mathias Aazami, MD_-Imam Reza University Hospital, Iran Oved Cohen, MD_Rambam Health Care Campus, Haifa, Israel

Vivek Pillai, MD_-Tirunal Institute for Medical Science and Technology, Trivandrum, India

Darshan Reddy, MD_University of Kwazulu, Natal, South Africa

2015 Surgical Robotics Fellowship Recipients

- Marisa Amaral, MD

- Diego Avella Patino, MD

- Erin Gillaspie, MD

- Onkar Khullar, MD

- Kiran Lagisetty, MD

- Gita Mody, MD

- Christopher Scott, MD

- Joanna Sesti, MD

- Kei Suzuki, MD

- Manuel Villa Sanchez, MD

- Anna Bendzsak, MD

- Matthew Bott, MD

- Marion Hochstetler, MD

- Johannes Kratz, MD

- Joseph Phillips, MD

- Eric Sceusi, MD

- Nestor Villamizar, MD

- Ory Wiesel, MD

- Jennifer Wilson, MD

- Moritz Wyler von Ballmoos, MD

F. Griffith Pearson Fellowship

Pablo Sanchez, MD-University of Maryland

James L. Lubawski, MD, Jr-Central DuPage Hospital

C. Walton Lillehei Resident Forum

Jeffrey E. Cohen, MD-Stanford University

Summer Intern Scholarship Recipients

- Chiaka Aribeana

- Adam Attaar

- Jassimran Bainiwal

- Eduardo Bent Robinson

- Emily Cerier

- Trevor Davis

- Whitney Faiella

- Kelsey Finkel

- Kelsey Gallo

- Rebecca Gao

- Brendan Heiden

- John Hennessey

- William Hollabaugh

- Lingling Huang

- Godly Jack

- Yeon Kim

- James Kostyla

- Katherine Leiby

- Ramon Li

- Peter Lo

- Jessica Luc
- Jessica Lucas

- Orestes Mavrothalassitis

- Alyssa Mazurek

- Lily Mei

- Rohan Menon

- Yafei Ouyang

- Neil Patel

- Golnaz Roshankar

- Evan Rotar

- Michael Rouse

- Paul Salazar

- Katherine Sanders

- Manoj Saraswat

- Shakir Saud

- Samuel Schnittman

- Yosef Schwartz

- Apollo Stack

- Soraiya Thura

- Richard Williams

- Sherry Wu 
Every Heartbeat Matters: Travel Awards for the 2015 AATS Cardiovascular Valve Symposium/Brazil

- Alvaro Albrecht, MD

- Laura Barboza, MD

- Leandro Batisti De Faria, MD

- César Benítez Pozo, MD

- Javier Brenes Gonzalez, MD

- Mariano Martin Candioti Lehmann, MD

- Roque Cordoba, MD

- Dilmar Cunha, MD

- Mauro De Sa, MD

- Thiago Dos Santos, MD

- Renato Faria, MD

- Christian Gonzalo Gil, MD

- Francisco Guzmán Aceituno, MD

- Gustavo Judas, MD

- Debora Klein Ferreira, MD

- Ricardo Liendo, MD

- Gabriel Longo, MD

- Evandro Carlos Lopes, MD

- Ignacio Lugones, MD

- Juan Navas Vasquez, MD

- Marco Antonio Praca Oliveira, MD

- Joao Oliveira, MD

- Cristiano Ourives, MD

- Gilberto Paulozzi Junior, MD

- Mauricio Fortuna Pinheiro, MD

- Fernando Platania, MD

- Virginia Graciela Rivas Arcia, MD

- Emma Salazar Barragán, MD

- Pedro Salerno, MD

- Lucia Salerno, MD

- Carlos Alberto Sancio Junior, MD

- Paulo Cesar Santos, MD

- Rodrigo Segalote, MD

- Renê Silva, MD

- Fabio Silva, MD

- Nicolas Zavaley, MD

\section{AATS Awards Applications}

Now Available at www.aats.org

July 2016 Deadlines

Third John Alexander Research Scholarship 2017-2019 provides an opportunity for research, training, and experience for North American surgeons committed to pursuing an academic career in cardiothoracic surgery.

- Research program must be undertaken within the first three years after completion of an approved North American cardiothoracic residency.

- Applications for the scholarship must be submitted during the candidate's first two years in an academic position.

- The scholarship will begin July 1, 2016, and conclude on June 30, 2018.

- The Scholarship provides an annual stipend of $\$ 80,000$ per year paid to the host institution for direct salary support and related research expenses.

Deadline: July 1, 2016

Evarts A. Graham Memorial Traveling Fellowship, 2017-2018 grants support for training of international surgeons who have been regarded as having the potential for later international thoracic surgical leadership.

- Candidate must be a non-North American who has not had extensive (exceeding a total of six months in duration) clinical training in North America prior to submitting an application.

- Candidate should have completed his/her formal training in general surgery and in thoracic and cardiovascular surgery, but should not have reached a senior position.

- The Fellowship provides a stipend of $\$ 75,000$ US, a major portion of which is intended for living and travel expenses incurred when visiting other medical centers in North America.

Deadline: July 1, 2016

\section{The Western Thoracic Surgical Association}

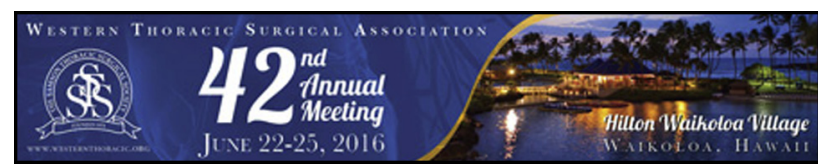

\section{WTSA 42nd Annual Meeting}

June 22-25, 2016

Hilton Waikoloa Village

Waikoloa, Hawaii

Visit the WTSA Web site at www.westernthoracic.org to: 